\title{
Hiperferritinemia em homens adultos e idosos: condições clínica e nutricional além da hemocromatose hereditária
}

\author{
Hyperferritinemia in adult and elderly men: clinical \\ condition beyond hereditary hemochromatosis
}

Mariluci Dos Santos Fortes', Caroline de Maman Oldra', Késia Zanuzo', Márcia Fernandes Nishiyama', Eloá Angélica Koehnlein’

'Universidade Federal da Fronteira Sul (UFFS) - Campus Realeza - Curso de Nutrição

E-mail para correspondência: Eloá Angélica Koehnlein - eloa.koehnlein@uffs.edu.br

\begin{abstract}
Resumo
Objetivo: Analisar características clínicas, bioquímicas, antropométricas e nutricionais de pacientes com hiperferritinemia e identificar a relação com sobrecarga de ferro associada à Hemocromatose Hereditária ou a relação com outros fatores. Métodos: Este estudo incluiu 27 homens, adultos e idosos, com hiperferritinemia, atendidos em uma clínica-escola de nutrição. Analisou-se dados sociodemográficos, clínicos, bioquímicos, antropométricos e do consumo alimentar. Para avaliação de sobrecarga de ferro por Hemocromatose Hereditária utilizou-se valores de Saturação de Transferrina >45\%; para Síndrome Metabólica, critérios da International Diabetes Federation e para o Fator Inflamatório Dietético, o software IF Ratings ${ }^{T M}$ of Common Foods (v. 32). Resultados: Foram observadas baixa frequência de sobrecarga de ferro associada à provável Hemocromatose Hereditária (11,11\%) e elevadas frequências de sedentarismo (44,44\%), etilismo (70,37\%), sobrepeso/obesidade (81,48\%), risco de doença cardiovascular (96,30\%), Síndrome Metabólica (40,74\%), Velocidade de Hemossedimentação alterada $(48,15 \%)$ e alterações bioquímicas. Observou-se dieta fortemente inflamatória, excessiva em energia, lipídios e ácidos graxos saturados e poliinsaturados, e insuficiente em fibras, vitaminas e minerais antioxidantes e ferro biodisponível para absorção, sendo esse último inversamente correlacionado com a ferritina sérica $(r=-0,540)$. Conclusão: Este estudo revelou baixa relação de hiperferritinemia associada à provável Hemocromatose Hereditária e elevada associação com alterações metabólicas. Destaca-se a importância da realização de estudos maiores que realizem a confirmação de Hemocromatose Hereditária por meio de teste genético e de Síndrome Dismetabólica de Sobrecarga de ferro por meio de biópsia hepática, bem como de estudos de intervenção, visando adequação dos hábitos alimentares e perfil metabólico destes pacientes.
\end{abstract}

Palavras-chave: Ferritina. Sobrecarga de Ferro. Hemocromatose. Obesidade. 


\begin{abstract}
Objective: Analyze clinical, biochemical, anthropometric and nutritional characteristics of patients with hyperferritinemia and identify the relationship with iron overload associated with Hereditary Hemochromatosis or the relation with other factors. Methods: This study included 27 men, adults and the elderly, with hyperferritinemia, attended in a nutrition school clinic. Were analyzed data sociodemographic, clinical, biochemical, anthropometric and food consumption. For evaluation of iron overload by Hereditary Hemochromatosis, were used values of Transferrin Saturation $>45 \%$; for the Metabolic Syndrome, were used criteria International Diabetes Federation and for the Dietary Inflammatory Factor, the software IF Ratings ${ }^{T M}$ of Common Foods (v. 32). Results: There was a low frequency of iron overload associated with the probable Hereditary Hemochromatosis (17,71\%) and high frequencies of sedentary lifestyle (44,44\%), alcoholism (70,37\%), overweight/obesity (87,48\%), risk of cardiovascular disease (96,30\%), Metabolic Syndrome (40,74\%), altered erythrocyte sedimentation rate (48,15\%) and biochemical alterations. It was observed a strongly inflammatory diet, excessive in energy, lipids and saturated and polyunsaturated fatty acids, and insufficient in fibers, vitamins and minerals antioxidant and iron bioavailable for absorption, the latter being inversely correlated with serum ferritin $(r=-0.540)$. Conclusion: This study revealed a low frequency of hyperferritinemia associated with probable Hereditary Hemochromatosis and a high association with metabolic factors. Highlights the importance of to carry out larger studies that perform the confirmation Hereditary Hemochromatosis by means of a genetic test and Dysmetabolic Syndrome of Iron Overload by means of liver biopsy, as well as of intervention studies, aiming to improve the dietary habits and metabolic profile of these patients.
\end{abstract}

Keywords: Ferritins. Iron Overload. Hemochromatosis. Obesity.

\title{
INTRODUÇÃO
}

A hiperferritinemia é frequentemente observada na prática clínica e, na maioria dos casos, seu diagnóstico acaba sendo correlacionado com a Hemocromatose Hereditária ( $\mathrm{HH})$, a qual é uma doença genética, caracterizada pelo aumento da absorção intestinal e acúmulo progressivo do ferro em diferentes órgãos do organismo ${ }^{1}$.

No entanto, estudos indicam que a hiperferritinemia não está mais relacionada somente a $\mathrm{HH}$, mas também a outros fatores, como processos inflamatórios e alterações metabólicas, sendo necessária a realização de estudos e investigações mais precisas para elucidá- $\operatorname{los}^{2}$.

Em vista disso, a hiperferritinemia associada a Síndrome Metabólica (SM) e a Doença hepática gordurosa não alcoólica (NAFLD) vem sendo estudada e descrita como Síndrome Dismetabólica de Sobrecarga de Ferro (DIOS). Estudos sugerem que 
a DIOS pode alterar o mecanismo de transporte do ferro e provocar um leve acúmulo de ferro no fígado, mantendo os valores de ferro sérico e de Saturação de Transferrina (ST) normais, diferente da $\mathrm{HH}$, na qual o acúmulo de ferro é maior e a ST apresenta valores superiores a $45 \% 3$.

Além da DIOS, também têm sido descrita na literatura, a Hiperferritinemia Dismetabólica (HFD) que difere da primeira condição, por não haver depósito de ferro, mas que estaria relacionada à resistência à insulina, e suas alterações metabólicas, por outro mecanismo 4 .

Desta forma, a ST no soro é um teste rápido e fidedigno para a avaliação da sobrecarga de ferro por $\mathrm{HH}$, sendo o parâmetro laboratorial mais importante e precoce para esse diagnóstico 5 . Por outro lado, a análise de características clínicas, bioquímicas, estado nutricional e consumo alimentar pode auxiliar na identificação de processos inflamatórios e alterações metabólicas e nutricionais que podem estar relacionadas a esta alteração ${ }^{5,6}$.

Diante do exposto, este estudo teve como objetivo analisar as características clínicas, bioquímicas, antropométricas e nutricionais de pacientes com hiperferritinemia, a fim de identificar a relação com sobrecarga de ferro associada à HH ou a relação com outros fatores.

\section{MATERIAIS E MÉTODOS}

O presente estudo possuiu caráter transversal com coleta de dados retrospectivos e foi realizado em uma clínica-escola de nutrição, localizada em um município de pequeno porte do Sudoeste do Paraná, que oferece atendimento nutricional gratuito. Foram selecionados todos os indivíduos atendidos no ano de 2016 que apresentaram hiperferritinemia (ferritina sérica > 300ng/mL para homens).

A amostra consistiu de 27 pacientes do gênero masculino, com idade entre 29 e 75 anos. Foram coletados, em um mesmo momento a partir dos prontuários de atendimento dos pacientes, dados sociodemográficos, clínicos, bioquímicos, antropométricos e do consumo alimentar.

Para avaliação bioquímica foram utilizados os valores de \% de ST, ferro sérico, Proteína C-Reativa (PCR), Velocidade de Hemossedimentação (VHS), glicose de jejum, Transaminase Glutâmica Oxalacética (TGO), Transaminase Glutâmica Pirúvica (TGP) 5 colesterol total, HDL-colesterol, LDL-colesterol e triglicerídeos 7 . 
Para associação da hiperferritinemia relacionada a sobrecarga de ferro por provável HH foram utilizados os valores de ST superiores a 45\%4. A SM foi identificada a partir dos critérios para diagnóstico estabelecidos pela International Diabetes Federation $(\mathrm{IDF})^{8}$.

A avaliação do estado nutricional foi realizada através dos dados de peso (Kg) e estatura (m) para cálculo do Índice de Massa Corporal (IMC), sendo classificado de acordo com os pontos de corte da Organização Mundial da Saúde ${ }^{9}$ para homens adultos com idade de 20 a 59 anos e segundo a classificação de Lipchitz ${ }^{10}$ para homens idosos com idade igual ou maior a 60 anos. Também avaliou-se o risco de Doença Cardiovascular (DCV) através dos indicadores de Circunferência da Cintura (CC) $(\geq 94 \mathrm{~cm})^{11}$ e Relação Cintura-Estatura (RCE) $(\geq 0,50)^{12}$.

A avaliação do consumo alimentar foi realizada através da avaliação de um Diário Alimentar de 3 dias (DA), sendo 3 dias não consecutivos e 1 dia obrigatoriamente referente ao final de semana ${ }^{13}$. Os dados coletados em medidas caseiras foram convertidos em gramas $(\mathrm{g})$ ou mililitros ( $\mathrm{ml}$ ) com o auxílio da Tabela de Medidas Referidas para os alimentos Consumidos no Brasil ${ }^{14}$. Foram avaliadas a ingestão de energia, macronutrientes, fibras, vitaminas e minerais antioxidantes (vitamina C, E e A, selênio, manganês, cobre e zinco) e ferro utilizando a Tabela Brasileira de Composição de Alimentos ${ }^{15}$ e a Tabela de Composição Nutricional dos Alimentos Consumidos no Brasil ${ }^{16}$.

A adequação do consumo alimentar de energia (Kcal) foi realizada conforme recomendação para perda de peso, de $20 \mathrm{Kcal} / \mathrm{Kg}$ de peso quando paciente apresentava sobrepeso/obesidade, e para manutenção do peso, de $25 \mathrm{Kcal} / \mathrm{Kg}$ de peso quando paciente apresentava-se eutrófico ${ }^{17}$. Para adequação do consumo de macronutrientes e fibras foram utilizadas as recomendações da Diretriz da Sociedade Brasileira de Cardiologia7 ${ }^{7}$ considerando a presença de comorbidades. Já a adequação do consumo de vitaminas e minerais foi realizada conforme as recomendações das DRIs, de acordo com as respectivas faixas etárias ${ }^{18,19}$.

A biodisponibilidade do ferro ingerido foi calculada através do método de Monsen et al. ${ }^{20}$ que leva em consideração o estoque corporal de ferro, a ingestão de ferro total, ferro heme, ferro não heme, vitamina $\mathrm{C}$ e a quantidade de carne por refeição. A partir do cálculo da biodisponibilidade de cada refeição foi calculado o percentual de absorção do ferro ingerido no dia, de cada paciente estudado, e as dietas foram classificadas em baixa, média ou alta biodisponibilidade ${ }^{21}$. 
Ainda, avaliou-se o Fator Inflamatório Dietético (FID) através do software IF Ratings $^{\mathrm{TM} 22}$, o qual classifica os alimentos em anti-inflamatórios, com valores de FID positivos, e inflamatórios, com valores de FID negativos. Para realização do cálculo, as medidas caseiras obtidas pelo DA foram transformadas em gramas e calculou-se o FID correspondente à quantidade em gramas de cada alimento consumido. A média do FID obtida nos três dias avaliados foi classificada em: Fortemente anti-inflamatória (200 ou superior); Moderadamente antiinflamatória (101 a 200); Ligeiramente anti-inflamatória (o a 100); Ligeiramente inflamatória (-1 a -100); Moderadamente inflamatória (-101 a -200) e Fortemente inflamatória (-201 ou inferior) ${ }^{23}$.

A avaliação do consumo de bebidas alcoólicas foi realizada com base nas informações relatadas pelos pacientes, sendo registrado o consumo aproximado em mililitros dos diferentes tipos de bebidas alcoólicas ingeridas nos diferentes dias da semana. Para o cálculo da quantidade de etanol, considerou-se o percentual de etanol da cerveja de $6 \%$, do vinho de $12 \%$ e dos destilados de $40 \%$, para a transformação do etanol em gramas foram multiplicados os mililitros pelo coeficiente de densidade do etanol de 0,8. A adequação do consumo de etanol foi realizada utilizando a recomendação de no máximo $30 \mathrm{~g}$ de etanol por dia, de preferência não habitualmente ${ }^{24}$.

A análise estatística foi realizada através de estatística descritiva, com médias e desvio padrão para variáveis contínuas e frequência relativa e absoluta para variáveis categóricas. Para a avaliação da associação entre variáveis utilizou-se o Coeficiente de Correlação de Spearman. Todas as análises estatísticas foram realizadas no programa Statistical Program for Social Sciences (SPSS), versão 19.0, sendo considerado nível de significância de $5 \%(\mathrm{p}<0,05)$.

O estudo foi aprovado pelo Comitê de Ética em Pesquisa com Seres Humanos da Universidade Federal da Fronteira Sul - UFFS com aprovação sob o CAAE No 41154814.7.0000.5564.

\section{RESULTADOS}

Do total de participantes avaliados, 74,07\% eram adultos e 25,93\% eram idosos. Com relação às características clínicas e antropométricas dos participantes do estudo, a maioria não apresentava histórico familiar de hiperferritinemia e quase metade já havia realizado flebotomia após orientação médica. Observou-se elevada 
frequência de sedentarismo e de doenças e sintomas associados, com destaque para o grupo de doenças metabólicas e alterações hepáticas (tabela 1).

Notou-se que $7,41 \%$ dos pacientes avaliados faziam uso de tabaco e verificou-se elevada frequência de consumo de bebidas alcoólicas acima do recomendado de $30 \mathrm{~g}$ de etanol por dia $(70,37 \%)$ (tabela 1$)$.

Quanto as características antropométricas dos pacientes, verificou-se elevada frequência de sobrepeso e obesidade $(81,48 \%)$ e de risco de DCV quando avaliado os indicadores de CC e RCE. Ainda, observou-se que 40,74\% dos pacientes apresentaram SM (tabela 1).

Tabela 1 - Características clínicas e antropométricas dos pacientes com hiperferritinemia de uma clínica-escola de nutrição do Sudoeste do Paraná (2016).

\begin{tabular}{|c|c|c|}
\hline Características & $\mathbf{n}$ & $\%$ \\
\hline \multicolumn{3}{|c|}{ Histórico familiar de hiperferritinemia } \\
\hline Sim & 3 & 11,11 \\
\hline Não & 24 & 88,89 \\
\hline \multicolumn{3}{|l|}{ Realização de flebotomia } \\
\hline Sim & 14 & 43,75 \\
\hline Não & 18 & 56,25 \\
\hline \multicolumn{3}{|c|}{ Doenças ou sintomas associados } \\
\hline Cardiovasculares & 10 & 10,31 \\
\hline Metabólicas & 39 & 40,20 \\
\hline Gastrointestinais & 6 & 6,19 \\
\hline Osteomusculares & 13 & 13,40 \\
\hline Alterações Hepáticas & 21 & 21,65 \\
\hline Outras & 8 & 8,25 \\
\hline \multicolumn{3}{|l|}{ Nível de atividade física } \\
\hline Sedentário & 12 & 44,44 \\
\hline Pouco ativo & 7 & 25,93 \\
\hline Ativo & 8 & 29,63 \\
\hline \multicolumn{3}{|l|}{ Tabagismo } \\
\hline Sim & 2 & 7,41 \\
\hline Não & 25 & 92,59 \\
\hline \multicolumn{3}{|l|}{ Consumo de etanol } \\
\hline Não & 5 & 18,52 \\
\hline Adequado & 3 & 11,11 \\
\hline Acima & 19 & 70,37 \\
\hline \multicolumn{3}{|c|}{ Estado Nutricional - IMC (Kg/m2) } \\
\hline Eutrofia & 5 & 18,52 \\
\hline Sobrepeso/ Excesso de Peso' & 13 & 48,15 \\
\hline Obesidade & 9 & 33,33 \\
\hline \multicolumn{3}{|l|}{ Circunferência da Cintura (cm) } \\
\hline Risco & 20 & 74,07 \\
\hline Sem risco & 7 & 25,93 \\
\hline \multicolumn{3}{|l|}{ Relação Cintura-Estatura } \\
\hline Risco & 26 & 96,30 \\
\hline Sem risco & 1 & 3,70 \\
\hline \multicolumn{3}{|l|}{ Síndrome Metabólica - IDF } \\
\hline Sim & 11 & 40,74 \\
\hline Não & 16 & 59,26 \\
\hline
\end{tabular}

Nota: 'Classificação para idosos.

n: Número absoluto; IMC: Índice de Massa Corporal.

A média de ferritina sérica encontrada foi de $682,01 \mathrm{ng} / \mathrm{mL}$, e analisando os valores de \% de ST e ferro sérico, observou-se que apenas 11,11\% e 3,70\%, respectivamente, 
apresentaram valores elevados nestes parâmetros. Por outro lado, quase metade dos pacientes apresentaram VHS elevada, o que está possivelmente relacionado a processos inflamatórios. Ainda, foi possível observar elevadas frequências de alterações nos valores de colesterol total, HDL, LDL, triglicerídeos, glicemia de jejum, TGO e TGP (Tabela 2).

Tabela 2 - Características bioquímicas dos pacientes com hiperferritinemia de uma clínica-escola de nutrição do Sudoeste do Paraná (2016).

\begin{tabular}{lcccc}
\hline Características & Média \pm DP & $\begin{array}{c}\text { Abaixo } \\
\mathbf{n}(\%)\end{array}$ & $\begin{array}{c}\text { Adequado } \\
\mathbf{n}(\%)\end{array}$ & $\begin{array}{c}\text { Elevado } \\
\mathbf{n}(\%)\end{array}$ \\
\hline ST (\%) & $36,17 \pm 8,21$ & 0 & $24(88,89)$ & $3(11,11)$ \\
Ferro Sérico (mcg/dL) & $116,37 \pm 26,12$ & 0 & $26(96,30)$ & $1(3,70)$ \\
PCR & -1 & 0 & $23(85,19)$ & $4(14,81)$ \\
VHS & -1 & 0 & $14(51,85)$ & $13(48,15)$ \\
Colesterol total(mg/dL) & $205,81 \pm 53,54$ & 0 & $12(44,44)$ & $15(55,56)$ \\
HDL-col (mg/dL) & $40,45 \pm 8,00$ & $17(62,96)$ & $10(37,04)$ & 0 \\
LDL-col (mg/dL) & $122,25 \pm 44,18$ & 0 & $16(59,26)$ & $8(29,63)$ \\
Triglicerídeos (mg/dL) & $224,95 \pm 161,74$ & 0 & $10(37,04)$ & $17(62,96)$ \\
Glicose jejum (mg/dL) & $106,18 \pm 40,82$ & $1(3,70)$ & $20(74,07)$ & $6(22,22)$ \\
TCO (U/L) & $52,95 \pm 50,26$ & 0 & $13(48,15)$ & $14(51,85)$ \\
TCP (U/L) & $65,43 \pm 60,30$ & 0 & $11(40,74)$ & $16(59,26)$ \\
\hline
\end{tabular}

Nota: 'A avaliação da PCR e VHS foi realizada com resultado negativo quando adequado e positivo quando elevado, não apresentando média e DP; ${ }^{2}$ Não foi possível calcular os valores de LDL-colesterol (mg/dL) de 3 pacientes $(11,11 \%)$ devido aos valores de triglicerídeos estarem superiores a $400 \mathrm{mg} / \mathrm{dL}$ (Sociedade Brasileira de Cardiologia, 2017).

DP: Desvio-Padrão; n: Número absoluto; ST: Saturação de Transferrina; PCR: Proteína C-Reativa; VHS: Velocidade de Hemossedimentação; TGO: Transaminase Glutâmica Oxalacética; TGP: Transaminase Glutâmica Pirúvica.

A avaliação do consumo alimentar dos pacientes demonstrou elevada frequência de consumo excessivo de energia, lipídios, ácidos graxos saturados e poliinsaturados, e consumo insuficiente de fibras, vitamina C, vitamina E, vitamina A, manganês, cobre e zinco. Em relação ao consumo de ferro da dieta, verificou-se que todos apresentaram consumo adequado, no entanto, as dietas apresentaram baixa biodisponibilidade de ferro, sendo que nenhum paciente apresentou dieta com alta biodisponibilidade (Figura 1). Por outro lado, avaliando o FID, todos os pacientes apresentaram dieta fortemente inflamatória, sendo o índice médio encontrado de $825,43 \pm 315,45$ (dados não apresentados em tabela). 
Figura 1 - Classificação da biodisponibilidade de ferro das dietas dos pacientes com hiperferritinemia de uma clínica-escola de nutrição do Sudoeste do Paraná (2016).

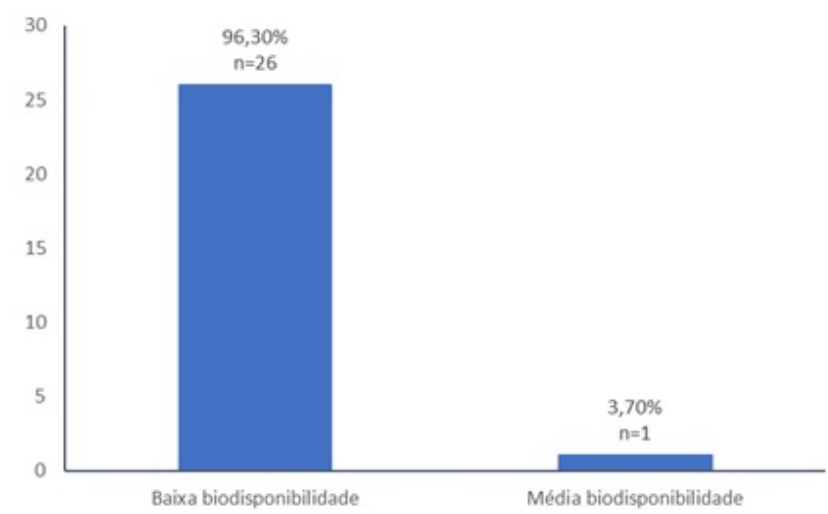

$y=$ número absoluto de pacientes; $x=$ Classificação das dietas em baixa e média biodisponibilidade conforme porcentagem de absorção do ferro.

Tabela 3 - Características do consumo alimentar dos pacientes com hiperferritinemia de uma clínica-escola de nutrição do Sudoeste do Paraná (2016).

\begin{tabular}{|c|c|c|c|c|}
\hline Características & Média \pm DP & $\begin{array}{c}\text { Consumo } \\
\text { Insuficiente } \\
\text { n (\%) }\end{array}$ & $\begin{array}{c}\text { Consumo } \\
\text { Adequado } \\
\text { n (\%) }\end{array}$ & $\begin{array}{c}\text { Consumo } \\
\text { Excessivo } \\
n(\%)\end{array}$ \\
\hline Energia (Kcal) & $2253,00 \pm 472,49$ & $4(14,81)$ & $5(18,52)$ & $18(66,67)$ \\
\hline Carboidratos (\%) & $51,79 \pm 7,64$ & $6(22,22)$ & $21(77,78)$ & 0 \\
\hline Proteínas (\%) & $16,44 \pm 2,46$ & 0 & $26(96,30)$ & $1(3,70)$ \\
\hline Lipídios (\%) & $31,76 \quad \pm 8,87$ & $3(11,11)$ & $12(44,44)$ & $12(44,44)$ \\
\hline $\operatorname{ACS}(\%)$ & $9,54 \pm 3,36$ & 0 & $8(29,63)$ & $19(70,37)$ \\
\hline $\operatorname{AGM}(\%)$ & $8,69 \pm 3,14$ & 0 & $27(100)$ & 0 \\
\hline AGP (\%) & $8,16 \pm 3,36$ & 0 & $19(70,37)$ & $8(29,63)$ \\
\hline Fibras (g) & $23,79 \pm 7,16$ & $15(55,56)$ & $12(44,44)$ & 0 \\
\hline Vitamina C (mg) & $94,95 \pm 64,75$ & $12(44,44)$ & $15(55,56)$ & 0 \\
\hline Vitamina E (mg) & $7,37 \pm 8,03$ & $22(81,48)$ & $5(18,52)$ & 0 \\
\hline Vitamina A $(\mu \mathrm{g})$ & $255,94 \pm 175,77$ & $25(92,59)$ & $2(7,41)$ & 0 \\
\hline Selênio ( $\mu g$ ) & $111,91 \pm 34,77$ & 0 & 27 (100) & 0 \\
\hline Manganês (mg) & $2,81 \pm 1,61$ & $15(55,56)$ & $12(44,44)$ & 0 \\
\hline Cobre (mg) & $1,46 \pm 0,76$ & $5(18,52)$ & $22(81,48)$ & 0 \\
\hline Zinco (mg) & $11,43 \pm 4,03$ & $7(25,93)$ & $20(74,07)$ & 0 \\
\hline Ferro (mg) & $11,44 \pm 2,61$ & 0 & $27(100)$ & 0 \\
\hline
\end{tabular}

DP: Desvio-Padrão; n: Número absoluto; ACS: Ácidos Graxos Saturados; AGM: Ácidos Graxos Monoinsaturados; AGP: Ácidos Graxos Poliinsaturados.

O FID foi obtido por meio de uma ferramenta desenvolvida por Reinagel ${ }^{23}$, que é o Inflammation Factor Rating System (IF Rating ${ }^{\mathrm{TM}}$ System). Essa ferramenta avalia 2400 alimentos em relação a 20 componentes, sendo considerados anti-inflamatórios: vitaminas A, C, E, B6, B12, K e folato, selênio, zinco, fitonutrientes, gorduras monoinsaturadas, ácido eicosapentaenóico (EPA), ácido docosahexaenóico (DHA) e ácido gama-linolênico (GLA), e sendo considerados inflamatórios: carga glicêmica, gorduras trans, gordura saturada, ácido araquidônico (ARA), e a relação dos ácidos 
graxos ômega 6/ômega 3. Esta ferramenta está disponível para utilização através de um aplicativo pago, o IF Tracker ${ }^{22}$.

Na Tabela 4 pode-se observar os resultados da avaliação da Correlação de Spearman, a qual mostrou correlação negativa moderada entre ferritina sérica e ferro biodisponível para absorção $(r=-0,540)$, entre ferro sérico e IMC $(r=-0,438)$ e ferro sérico e CC $(r=-0,433)$ e correlação negativa fraca entre ferro sérico e RCE $(r=-$ $0,394)$. Ainda, foi possível observar correlação negativa fraca entre \% de ST e CC ( $r=-$ $0,384)$ e correlação negativa moderada entre \% de ST e triglicerídeos $(r=-0,445)$ e entre \% de ST e TGP $(r=-0,417)$. Para todas as correlações foi considerado nível de significância de $5 \%(\mathrm{p}<0,05)$.

Tabela 4 - Correlação das variáveis antropométricas, dietéticas e bioquímicas com ferritina sérica, ferro sérico e saturação de transferrina dos pacientes com hiperferritinemia de uma clínica-escola de nutrição do Sudoeste do Paraná (2016)

\begin{tabular}{lccc}
\hline Variáveis & $\begin{array}{c}\text { Ferritina sérica } \\
\text { (ng/mL) } \\
\text { Correlação (p) }\end{array}$ & $\begin{array}{c}\text { Ferro sérico } \\
\text { (mcg/mL) } \\
\text { Correlação (p) }\end{array}$ & $\begin{array}{c}\text { ST } \\
\text { (\%) }\end{array}$ \\
\hline Correlação (p)
\end{tabular}

Nota: 'A correlação foi realizada através do teste de Correlação de Spearman. Valor de $p<0,05$ foi considerado significativo; ${ }^{2}$ Estimativa com base na biodisponibilidade de ferro da dieta.

ST: Saturação de Transferrina; IMC: Índice de Massa Corporal; CC: Circunferência da Cintura; RCE: Relação CinturaEstatura; IID: Índice Inflamatório Dietético; TCP: Transaminase Glutâmica Pirúvica.

\section{DISCUSSÃO}

O perfil de idade e ferritina sérica dos pacientes avaliados foi semelhante ao encontrado em estudo realizado por Biasoli et al. ${ }^{1}$ que avaliou uma amostragem de 5306 indivíduos e também observou maior prevalência de hiperferritinemia no gênero masculino, entre 20 e 60 anos, com média de ferritina de 616,17 ng/mL.

A frequência de hiperferritinemia possivelmente associada a HH foi baixa (11,11\%), estando de acordo com estudos que descrevem que apenas $10 \%$ dos casos de hiperferritinemia são devido a este fator ${ }^{2}$. O valor de ST acima de $45 \%$ é o parâmetro laboratorial mais importante e precoce para o diagnóstico de sobrecarga de ferro por 
HH. No entanto, é necessária a realização da pesquisa das mutações do gene HFE (C282Y, H63D e S65C) para a confirmação desse diagnóstico ${ }^{25}$, sendo essa uma limitação do presente estudo.

Sabe-se que a ferritina sérica pode estar elevada sem nenhuma relação com a sobrecarga de ferro, sendo observado nestes casos valores de ST normais ${ }^{25}$. Estudos indicam que isso ocorre em $90 \%$ dos casos de hiperferritinemia, os quais estão relacionados a outros fatores, como doenças crônicas, processos inflamatórios, diabetes, doenças hepáticas, obesidade, etilismo e $\mathrm{SM}^{2,25}$ fatores estes observados com elevada frequência neste estudo.

As frequências de SM observadas nos pacientes avaliados estão de acordo com estudos que indicam que a SM está fortemente associada com a hiperferritinemia, como demonstrado por Rauber ${ }^{26}$, que analisando 94 pacientes com SM, observaram que $27,7 \%$ apresentavam hiperferritinemia. No entanto, a associação entre hiperferritinemia, SM e a NAFLD vem sendo descrita mais recentemente como DIOS ou HFD. Estudos indicam que SM, esteatose, resistência à insulina e predisposição genética podem alterar o mecanismo de transporte do ferro e gerar sobrecarga hepática de ferro, necessitando de maiores estudos para esclarecer essa alteração. Desta forma, nos casos de DIOS, os valores de ferro sérico e de ST permanecem normais ou suavemente elevados e o fígado pode apresentar um leve acúmulo de ferro, diferente da $\mathrm{HH}$ onde o acúmulo é maior ${ }^{3}$. Nestes casos, a confirmação do diagnóstico do acúmulo de ferro pode ser realizada por meio de biópsia hepática ${ }^{25}$, sendo essa outra limitação do presente estudo, no qual não foi realizada.

Observou-se correlação inversa moderada e fraca entre ferro sérico e IMC ( $\mathrm{r}=-$ $0,438)$, CC $(r=-0,433)$ e RCE $(r=-0,394)$, enquanto a \% de ST foi inversamente correlacionada com a CC $(r=-0,384)$, triglicerídeos $(r=-0,445)$ e TGP $(r=-0,417)$. Estes dados indicam que o aumento da ST não se relacionou com alterações metabólicas no que diz respeito ao acúmulo central de gordura, hipertrigliceridemia e alteração hepática, indicando que a sobrecarga de ferro associada a HH parece não ser frequente em pacientes com alterações associadas a SM e DIOS.

Embora pesquisas apontem os benefícios da redução do ferro através de flebotomia, a orientação desta conduta deve ser realizada com cautela de maneira individualizada, apenas para pacientes com confirmação de sobrecarga de ferro por HH ou DIOS. No entanto, é comum na prática clínica a realização deste procedimento sem essa confirmação, como foi observada a elevada frequência neste estudo. Da 
mesma forma, a orientação para restrição de alimentos fontes de ferro, inserção de alimentos inibidores da absorção do ferro e administração de medicamentos quelantes deve ser realizada apenas para este mesmo grupo ${ }^{27}$, visto que estas podem causar restrições alimentares importantes, como observado neste estudo, que a maioria dos pacientes apresentaram dieta com baixa biodisponibilidade de ferro, sendo que o ferro biodisponível para absorção foi inversamente correlacionado com os valores de ferritina sérica dos pacientes $(r=-0,540)$.

Estudo realizado por Leite; Rocha; Brandão-Neto ${ }^{28}$, através da análise de 180 artigos, fortaleceu a proposição da obesidade enquanto doença inflamatória, através do aumento de adipocinas pró-inflamatórias no tecido adiposo, como o TNF- $\alpha$, IL-6, PCR, MCP-1, leptina e resistina, assim como, fortaleceu a associação da inflamação com o surgimento ou agravamento das comorbidades da obesidade, em especial diabetes tipo 2 e doenças cardiovasculares. Nesse sentido, sabe-se do papel da ferritina como mediador pró-inflamatório, a qual desempenha papel na indução da expressão de moléculas inflamatórias, com destaque para a L-ferritina que pode apresentar efeito estimulador sobre a proliferação celular, independente da disponibilidade de ferro ${ }^{28}$.

Neste estudo, observou-se baixa prevalência de indícios de processos inflamatórios quando avaliado os valores de PCR, a qual é classificada como uma das principais proteínas de fase aguda de processos inflamatórios. No entanto, quase metade dos pacientes apresentaram indícios de processos inflamatórios quando avaliado os valores de VHS, a qual também tem sido utilizada como um marcador da resposta inflamatória, por encontrar-se elevada em condições de elevação de proteínas inflamatórias de fase aguda de peso molecular elevado 5 .

Inúmeras evidências indicam que os hábitos alimentares desempenham papel central na regulação da inflamação crônica, sendo que pacientes portadores de Doenças Crônicas Não Transmissíveis (DCNT) apresentam relação entre a inflamação e o padrão alimentar. Dentre os fatores envolvidos, destaca-se a ingestão excessiva de calorias e macronutrientes, os quais possuem potencial para causar estresse oxidativo, estimulando os marcadores de inflamação. Ainda, estudos demonstram que o desequilíbrio entre as quantidades de gordura, peso corporal, lipoproteínas e lipídios em pacientes com obesidade aumentam a suscetibilidade do organismo a lesões oxidativas, também influenciando na ocorrência de processos inflamatórios. Por outro lado, hábitos alimentares compostos de alimentos ricos em 
componentes bioativos estão inteiramente relacionados com o controle e/ou a prevenção de múltiplas inflamações. As vitaminas e minerais antioxidantes possuem papel decisivo na modulação do processo inflamatório, pois são capazes de sequestrar radicais livres, reduzindo ou impedindo os danos causados por essas moléculas, como a oxidação e inflamação de diversas células e tecidos ${ }^{29}$.

No entanto, através da análise do FID observou-se que todos os pacientes apresentaram dieta fortemente inflamatória $(-825,43 \pm 315,45)$, resultado este que parece refletir as inadequações observadas na análise do consumo alimentar de macronutrientes, fibras, vitaminas e minerais antioxidantes. Estes dados estão de acordo com estudo realizado por Pimentel; Santos; Neto $^{30}$ que analisando a dieta de 74 pacientes nefropatas, com presença de comorbidades como as observadas neste estudo, encontraram elevadas frequências de inadequação no consumo de macronutrientes, fibras, vitaminas e minerais antioxidantes, assim como, 44,4\% apresentaram dieta fortemente inflamatória e 19,4\% dieta inflamatória.

Costa dos Santos et al. ${ }^{31}$ verificaram em estudo realizado com 296 homens adultos residentes em Fortaleza-CE, que o FID mostrou-se diretamente relacionado à obesidade, avaliada por meio do IMC, CC, RCE, Razão Cintura/Quadril e porcentagem de gordura corporal.

Estudo realizado por Ruiz-Canela, Bes-Rastrollo, Martínez-González ${ }^{32}$, analisando vários estudos que investigaram a associação entre o potencial inflamatório da dieta com fatores de risco cardiometabólicos e doenças cardiovasculares, demonstrou o papel da inflamação como um mecanismo envolvido no processo fisiopatológico de muitas doenças crônicas, sendo que, vários estudos demonstraram consistentemente uma associação direta entre dieta pró-inflamatória e maior risco de DCV, SM e mortalidade geral.

\section{CONCLUSÃO}

Os resultados do presente estudo sugerem para a amostra avaliada, baixa relação de hiperferritinemia com sobrecarga de ferro associada à provável HH e elevada associação de outros fatores à hiperferritinemia, como alterações metabólicas e nutricionais, risco de doença cardiovascular, processos inflamatórios, elevado consumo de bebidas alcoólicas e dieta fortemente inflamatória, com baixo consumo de vitaminas e minerais antioxidantes. 
Desta forma, destaca-se a importância da realização de estudos com grupos maiores e que realizem a confirmação destes diagnósticos, para melhor avaliar os fatores envolvidos na hiperferritinemia. Ainda, destaca-se a importância da realização de estudos de intervenção, que visem a adequação dos hábitos alimentares destes pacientes, através de orientação para o consumo de alimentos anti-inflamatórios e vitaminas e minerais antioxidantes, com o intuito de reduzir o efeito inflamatório da dieta e obter melhorias no estado nutricional e perfil metabólico destes pacientes.

\section{Agradecimento}

À Fundação Araucária pelo apoio financeiro, através do programa de bolsas de iniciação científica (PIBIC), edital no 593/UFFS/2016.

\section{REFERÊNCIAS}

1. Biasoli R, et al. Avaliação da hiperferritinemia numa amostragem de 5306 casos: as médias encontradas foram clinicamente significativas. Rev Bras Hematol Hemoter. 2012;34(Supl. 2):89-326.

2. Goot K, et al. Elevated serum ferritin - What should GPs know? Australian Family Physician. 2012;41(12):945-949.

3. Bardou-Jacquet $E$, et al. Long- term course after initial iron removal of iron excess in patients with dysmetabolic iron overload syndrome. Eur J Gastroenterol Hepatol. 2014;26(4):418-21.

4. Datz C, Muller E, Aigner E. Iron overload and non-alcoolic fatty liver disease. Minerva endocrinol 2017;42(2):173-83.

5. Calixto-Lima L, Reis NT. Interpretação de exames laboratoriais aplicados à nutrição clínica. $1^{\mathrm{a}}$ ed. Rio de Janeiro: Rubio. 2012.

6. Pedraza DF, Menezes TM. Questionários de Frequência de Consumo Alimentar desenvolvidos e validados para população do Brasil: revisão da literatura. Ciência \& Saúde Coletiva. 2015;20(9):2697-2720.

7. Sociedade Brasileira de Cardiologia. Atualização da Diretriz Brasileira de Dislipidemias e prevenção da Aterosclerose. Arq Bras Cardiol. 2017;109(2Supl.1):1-76.

8. International Diabetes Federation (IDF). Metabolic Syndrome Definition. The International Diabetes Federation (IDF) consensus worldwide definition of the metabolic syndrome. 2006; 1-16. Disponível em: <https://www.idf.org/elibrary/consensus-statements/60-idfconsensus-worldwide-definitionof-themetabolic-syndrome>. Acessado em: 03 de set. de 2018.

9. World Health Organization (WHO). Obesity: preventing and managing the global epidemic of obesity. Report of the WHO Consultation of Obesity. Geneva: World Health Organization. 1997. Disponível em: <http://www.who.int/nutrition/publications/obesity/WHO_TRS_894/en/>. Acessado em: 03 de set. de 2018. 
10. Lipschitz DA. Screening for nutritional status in the elderly. Prim Care. 1994;21(1):55-67.

11. World Health Organization (WHO). Obesity: preventing and managing the global epidemic. Geneva: World Health Organization. 1998. Disponível em: <http://www.who.int/nutrition/publications/obesity/WHO_TRS_894/en/>. Acessado em: 03 de set. de 2018.

12. Browning LM, Hsieh SD, Ashwell M. A systematic review of waist-to-height ratio as a screening tool for the prediction of cardio - vascular disease and diabetes: 0.5 could be a suitable global boundary value. Nutr Res Rev. 2010; 23(2):247-69.

13. Rossi L, Caruso L, Galante AP. Avaliação nutricional: novas perspectivas. São Paulo: Roca, 2008.

14. IBGE - Instituto Brasileiro de Geografia e Estatística. Pesquisa de Orçamentos Familiares - POF. Tabela de medidas referidas para os alimentos consumidos no Brasil. Rio de Janeiro, 2011. Disponível em: <https://biblioteca.ibge.gov.br/visualizacao/livros/liv50000.pdf>. Acessado em: 03 de set. de 2018.

15. Tabela Brasileira de Composição de Alimentos - TACO. 4. Ed. Campinas: NEPAUNICAMP, 2011. Disponível em: <http://www.cfn.org.br/index.php/tabelabrasileira-de-composicao-de-alimentos-4a-edicao/>. Acessado em: 03 de set. de 2018.

16. IBGE - Instituto Brasileiro de Geografia e Estatística. Pesquisa de Orçamentos Familiares - POF. Tabela de composição nutricional dos alimentos consumidos no Brasil. Rio de Janeiro, 2011. Disponível em: <https://biblioteca.ibge.gov.br/visualizacao/livros/liv50002.pdf>. Acessado em: 03 de set. de 2018.

17. Guimarães AF, Galisa MS. Cálculos Nutricionais: Conceitos e aplicações práticas. São Paulo: M.Books do Brasil Editora Ltda, 2008.

18. Institute of Medicine. Dietary reference intakes for vitamin C, vitamin E, selenium, and carotenoids. Washington, DC: National Academy Press; 2000.

19. Institute of Medicine. Dietary reference intakes for vitamin A, vitamin K, arsenic, boron, chromium, copper, iodine, iron, manganese, molybdenum, nickel, silicon, vanadium, and zinc. Washington, DC: National Academy Press; 2001.

20. Monsen ER, Hallberg L, Layrisse M, Hegsted DM, Cook JD, Mertz W, et al. Estimation of available dietary iron. The American Journal of Clinical Nutrition. 1978;31(1):134-141.

21. World Health Organization (WHO). Guidelines on food fortification with micronutrients. Geneva: WHO, 2006. Disponível em: $<$ www.who.int/nutrition/publications/guide_food_fortification_micronutrient s.pdf $>$. Acessado em: 03 de set. de 2018.

22. IF Rating. Home of the IF Rating system. [acessado em 2019 Set 9] Disponível em: https://inflammationfactor.com/.

23. Reinagel M. The Inflammation-Free Diet Plan. 1. McGraw-Hill; 2006.

24. Sociedade Brasileira de Cardiologia. VI Diretrizes Brasileiras de Hipertensão Arterial. Arq Bras Cardiol. 2010;95(1 supl.1):1-51. 
25. Cançado RD, Chiattone CS. Visão atual da hemocromatose hereditária. Rev Bras Hematol Hemoter. 2010;32(6):469-475.

26. Rauber MR. Avaliação molecular e bioquímica do metabolismo do ferro em pacientes portadores de Síndrome Metabólica. [dissertação]. Porto Alegre: Universidade Federal do Rio Grande do Sul; 2014. Disponível em: <http://hdl.handle.net/10183/131209>. Acessado em: 03 de set. de 2018.

27. Beaton MD, Adams PC. Treatment of hyperferritinemia. Ann Hepatology. 2012;11(3):294-300.

28. Leite LD, Rocha EDM, Brandão-Neto J. Obesidade: uma doença inflamatória. Rev Ciência \& Saúde. 2009;2(2):85-95.

29. França BK, et al. Peroxidação lipídica e obesidade: Métodos para aferição do estresse oxidativo em obesos. Jornal Português de Gastrenterologia. 2013;20(5):199-206.

30. Pimentel CKS, Santos CM, Neto LML. Características nutricionais e fator inflamatório das dietas usadas por pacientes em terapia renal substitutiva. BRASPEN J. 2016;31(3): 208-12.

31. Santos CC, Machado SP, Nogueira VC, Sampaio HAC, Farias BO, Vieira LCO. O fator inflamatório dietético está diretamente relacionado aos indicadores antropométricos de obesidade em trabalhadores de uma indústria metalúrgica. Nutrición Clínica y Dietética Hospitalaria. 2019; 39(3):22-29.

32. Ruiz-Canela M, Bes-Rastrollo M, Martínez-González MA. The Role of Dietary Inflammatory Index in Cardiovascular Disease, Metabolic Syndrome and Mortality. Int J Mol Sci. 2016;17(8):1265.

Submissão: 14/06/2020

Aprovação: 17/03/2021 